\title{
Die wissenschaftstheoretische Begründung der Medizin als Naturwissenschaft bei Hermann von Helmholtz (1821-1894)
}

Josef N. Neumann

\section{SUMMARY}

In this study an attempt will be made to discuss the epistemological problems in the theory and practice of modern technical medicine in the writings of Hermann von Helmholtz. An inquiry into the relationship between von Helmholtz' thinking and the critical philosophy of Immanuel Kant is followed by the characteristics of von Helmholtz" philosophy of science which he himself called "empirical theory". The question of medicine as a science finally leads to the main problem of medical epistemology, viz., the relationship between theoretical knowledge and practice in medicine. In this context the anthropological dimension is brought into consideration.

\section{ZuSAMMENFASSUNG}

In der hier vorgelegten Studie wird der Versuch unternommen, ausgehend von einer Analyse der Schriften von Hermann von Helmholtz grundsätzliche wissenschaftstheoretische Probleme der naturwissenschaftlich-technischen Medizin darzustellen. Dabei wird zunächst die Frage gestellt nach der Bedeutung von Kants kritischer Philosophie im wissenschaftstheoretischen Denken von Hermann von Helmholtz. In einem weiteren Schritt wird v. Helmholtz' medizinische Wissenschaftstheorie, die er selbst «empiristische Theorie» nennt, dargestellt. Die Erörterung der Frage, auf welche Weise v. Helmholtz die Medizin als «Naturwissenschaft» begründet, führt schliesslich zum Hauptproblem der medizinischen Wissenschaftstheorie, der Bestimmung des Verhältnisses von naturwissenschaftlicher Erkenntnis und medizinischer Praxis. In diesem Zusammenhang ist besonders nach dem Menschenbild in der naturwissenschaftlichtechnischen Medizin zu fragen. 
In der Vorrede zur dritten Auflage (1884) seiner «Vorträge und Reden» erinnert sich v. Helmholtz:

«Ich war im Beginne meiner Laufbahn ein gläubigerer Kantianer als ich jetzt bin; oder vielmehr, ich glaubte damals, dass das, was ich bei Kant geändert zu sehen wünschte, unerhebliche Nebenpunkte wären, welche neben dem, was ich noch jetzt als eine Hauptleistung hochschätze, nicht in Betracht kämen, bis ich später gefunden habe, dass sich die stricten Kantianer der jetzigen Periode hauptsächlich da festheften und da die höchste Entwicklung des Philosophen sehen, wo Kant meines Erachtens die ungenügenden Vorkenntnisse seiner Zeit und namentlich ihre metaphysischen Vorurteile nicht ganz überwunden und das Ziel, welches er sich gesteckt hatte, nicht ganz erreicht hat.» ${ }^{1}$

Von Helmholtz macht deutlich, dass sich sein Verhältnis zur Kantischen Philosophie im Werdegang seines wissenschaftlichen Denkens gewandelt hat. Dennoch blieb er von der unverzichtbaren Bedeutung der Philosophie für die Begründung wissenschaftlichen Erkennens und theoretisch fundierten Handelns auch dann überzeugt, als er seit den 80er Jahren des 19. Jahrhunderts als der führende Repräsentant der auf technische Anwendbarkeit ausgerichteten naturwissenschaftlichen Forschung in Deutschland galt und 1888 erster Präsident der von Werner von Siemens geförderten Physikalischen Reichsanstalt wurde. In der «akademischen Festrede» am 22.11.1862 in Heidelberg, in der er sich allgemein mit dem Verhältnis von Philosophie und Naturwissenschaften beschäftigte, machte v. Helmholtz deutlich, dass seine Hochschätzung vor allem Kants kritischer Philosophie, nicht hingegen Hegels dialektischem System und dem deutschen Idealismus gilt, den v. Helmholtz mitverantwortlich macht für eine gewisse Entfremdung, die im 19. Jahrhundert zwischen Philosophie und Naturwissenschaften eingetreten sei. Er sagt:

«Ein solcher Gegensatz ist in der Tat eine Zeitlang fühlbar gewesen und scheint mir namentlich unter dem Einfluss der Hegelschen Philosophie sich entwickelt zu haben oder durch diese Philosophie mindestens klarer als vorher an das Licht gezogen worden zu sein. Denn am Ende des vorigen Jahrhunderts unter dem Einflusse der Kantschen Lehre war eine solche Trennung noch nicht ausgesprochen, diese Philosophie stand vielmehr mit den Naturwissenschaften auf genau gleichem Boden, wie am besten Kants eigene naturwissenschaftliche Arbeiten zeigen [...] Kants kritische Philosophie ging nur darauf aus, die Quellen und die Berechtigung unseres Wissens zu prüfen und den einzelnen übrigen Wissenschaften gegenüber den Massstab für ihre geistige Arbeit aufzustellen.» ${ }^{2}$

Wenn hier Philosophie auch ausschliesslich als wissenschaftstheoretische Methodenkritik bestimmt wird, ein reduktionistischer Philosophiebegriff, dem Kant nicht zugestimmt hätte und dessen Berechtigung zu untersuchen 
sein wird, so kann dennoch gesagt werden, dass v. Helmholtz' Verständnis von Naturwissenschaft und «Hinwendung» der Medizin zum naturwissenschaftlichen Denken nicht «Abwendung» von der Philosophie bedeutet. Durch seinen Vater, Ferdinand Helmholtz, Gymnasialprofessor für Deutsch und Griechisch in Potsdam, «der einen tiefen Eindruck von Fichte's Idealismus behalten hatte» und den er «mit Collegen, die Hegel oder Kant verehrten, oft habe streiten hören ${ }^{3}$, wurde das Interesse an erkenntnistheoretischen Fragen früh geweckt.

W. Israel unterscheidet in v. Helmholtz' philosophischem Denken zwei Perioden: vor $1855^{4}$ orientiere er sich eng an Kants Erkenntnistheorie und transzendentaler Methode; in der Zeit danach sei ein deutlicher Einfluss der englischen Empiristen festzustellen, der wesentlich zu dem erkenntnistheoretischen Konzept beigetragen habe, das v. Helmholtz selbst «empiristische Theorie» nannte ${ }^{5}$. Während Israel die Meinung vertritt, dass die Entwicklung der v. Helmholtzschen Erkenntnistheorie besonders dadurch gekennzeichnet sei, dass in der Hypothesenbildung der Induktion immer grössere Bedeutung im Vergleich zur Deduktion beigemessen werde, versuchen Hörz und Wollgast in einer differenzierten Studie zu zeigen, dass es nicht gerechtfertigt ist, v. Helmholtz' philosophisches Denken einer der philosophiehistorischen Richtungen seiner Zeit zuzuordnen. Im Begriff «Zeichen» für das in der sinnlichen Wahrnehmung Gegebene anerkennt v. Helmholtz Kants Unterscheidung von «Ding an sich» und «Erscheinungsding» und hält damit Distanz sowohl zum Positivismus als auch zur nativistischen Theorie der Raumanschauung, «in welcher die Annahme gemacht wird, dass die Netzhaut sich selbst in ihrer räumlichen Ausdehnung empfinde, und bestimmte Raumvorstellungen vermittels eines angeborenen Mechanismus entstehen, dass also auch die specielle Lokalisation jedes Eindruckes durch die unmittelbare Anschauung gegeben ist [...].» ${ }^{6}$ Hörz und Wollgast bezeichnen die «empiristische Theorie» als «naturwissenschaftlich begründete Theorie der Abbildung ${ }^{7}$; ihr differenzierender Vergleich mit den philosophischen Strömungen des 19. Jahrhunderts findet aber dort seine Grenze, wo die genannten Autoren v. Helmholtz' Erkenntnistheorie als «naturwissenschaftlichen Materialismus» etikettieren und als dialektische Vorstufe des weltanschaulichen Materialismus zu vereinnahmen versuchen.

Es ist problematisch, einen Physiker und Physiologen als «Materialisten» zu bezeichnen, dessen Erkenntnistheorie bis zuletzt eine philosophische Auseinandersetzung mit Kants transzendentaler Methode war. Von daher steht in den folgenden Ausführungen nicht die Frage einer philosophie- 
historischen oder weltanschaulichen Zuordnung im Vordergrund. Es soll vielmehr erstens v. Helmholtz' philosophisches Denken in seinem Verhältnis zu Kants kritischer Philosophie bestimmt werden; zweitens ist die «empiristische Theorie» in ihren Grundzügen darzustellen, damit drittens das Verhältnis von Physik und Physiologie bestimmt und auf diese Weise präzisiert werden kann, was «Hinwendung der Medizin zu den Naturwissenschaften» in v. Helmholtz' Verständnis bedeutet.

\section{Von Helmholtz' Verhältnis zu Kants kritischer Philosophie}

An seinem 70. Geburtstag (1891) erinnerte sich v. Helmholtz an den Beginn seiner wissenschaftlichen Arbeit:

«Auch war in der That das Erste, was mich fesselte, vorzugsweise die geistige Bewältigung der uns anfangs fremd gegenüberstehenden Natur durch die logische Form des Gesetzes. Aber natürlich schloss sich bald die Erkenntniss an, dass die Kenntniss der Gesetze der Naturvorgänge auch der Zauberschlüssel sei, der seinem Inhaber Macht über die Natur in die Hände gebe. In diesen Gedankenkreisen fühlte ich mich heimisch.» ${ }^{8}$

Im Nachlass fand sich der Entwurf einer Rede mit dem Titel, «Über dauernde Bewegungsformen und scheinbare Substanzen», die v. Helmholtz 1894 auf der Naturforscherversammlung in Wien hatte vortragen wollen. Darin stellt sich v. Helmholtz nach Königsbergers Urteil «ganz auf den Standpunkt von Kant, der die Substanz nicht in den Dingen, sondern in den unveränderlichen Beziehungen sucht, welche an die Stelle der Dinge treten, und so sind ihm Substanzen jene oben definierten Integrationskonstanzen, welche dauernde Bewegungsformen darstellen, aber im Sinne der materiellen Substanzen nur scheinbare sind.» ${ }^{9}$

Die beiden Hinweise verdeutlichen, dass v. Helmholtz' erkenntnistheoretisches Denken sich durchgehend an Kants kritischer Philosophie orientierte. Mit dieser hat er sich besonders in zwei Arbeiten auseinandergesetzt, in der früheren Schrift «Über das Sehen des Menschen» (1855) und später in der Rede «Die Thatsachen in der Wahrnehmung» (1878), so dass die Entwicklung seines philosophischen Denkens im Verhältnis zu Kant in einem Vergleich verfolgt werden kann.

In v. Helmholtz' erkenntnistheoretischen Arbeiten ist durchgängig der Hinweis zu finden, dass «das Wirkliche», «was hinter dem Wechsel der Erscheinungen stehend auf uns einwirkt», das Naturgesetz sei. Von daher habe Naturwissenschaft die Aufgabe, «die letzten unveränderlichen Ursa- 
chen der Vorgänge in der Natur aufzufinden.» ${ }^{10}$ Auch der Begriff «Substanz», das, «was ohne Abhängigkeit von Anderem gleich bleibt in allem Wechsel der Zeit», wird nicht von der Materie aus bestimmt; denn «eine reine Materie wäre für die übrige Natur gleichgültig, weil sie nie eine Veränderung in dieser oder in unseren Sinnesorganen bedingen könnte; ...» Das Wirkliche ist das, was Veränderung bewirkt und selbst gleich bleibt. Substanz ist also «das gleichbleibende Verhältniss zwischen veränderlichen Grössen : das sie verbindende Gesetz». ${ }^{11}$

Hinter diesen grundlegenden Begriffsbestimmungen steht Kants Unterscheidung von «Erscheinungsding» und «Ding an sich» von der v. Helmholtz auch in der «empiristischen Theorie» nicht abgewichen ist und die er aus physiologischer Sicht durch die Lehre von den spezifischen Sinnesenergien als bestätigt ansah. Sein Lehrer Johannes Müller hatte die Subjektivität aller empirischen Erkenntnis aufgezeigt, nach der Art und Qualität unserer Empfindungen, ob sie Licht, Wärme, Ton oder Geschmack seien, ebenso von der Natur unserer Sinnesnerven abhängen, wie von den äusseren Gegenständen, ${ }^{12}$ und Kant hatte in der Kritik der reinen Vernunft Raum und Zeit als die der Erfahrung vorausgehenden Formen der Anschauung und die Kategorien als reine Verstandesbegriffe bestimmt, die nicht durch empirische Erkenntnis gewonnen sind, diese aber als Denkformen a priori ermöglichen. So urteilt v. Helmholtz:

«Gerade dasselbe, was in neuerer Zeit die Physiologie der Sinne auf dem Wege der Erfahrung nachgewiesen hat, suchte Kant schon früher für die Vorstellungen des menschlichen Geistes überhaupt nachzuweisen, indem er den Antheil darlegte, welchen die besonderen eingeborenen Gesetze des Geistes, gleichsam die Organisation des Geistes, an unseren Vorstellungen haben.» ${ }^{13}$

Im Gesetz der spezifischen Sinnesenergien sieht v. Helmholtz die naturwissenschaftliche Bestätigung der Kantschen Erkenntnistheorie und trifft von daher zwischen Philosophie und Naturwissenschaften die Unterscheidung:

«Die erstere, welche die geistige Seite betrachtet, sucht aus unserem Wissen und Vorstellen auszuscheiden, was aus den Einwirkungen der Körperwelt herrührt, um rein hinzustellen, was der eigenen Thätigkeit des Geistes angehört. Die Naturwissenschaft im Gegentheil sucht abzuscheiden, was Definition, Bezeichnung, Vorstellungsform, Hypothese ist, um rein übrig zu behalten, was der Welt der Wirklichkeit angehört, deren Gesetze sie sucht.» ${ }^{14}$

Weil der Mensch nicht in der Lage ist, die Dinge zu erkennen, wie sie «wirklich», d.h. unabhängig vom Beobachter und dessen physischer und psychischer Bedingtheit sind, hält v. Helmholtz eine kritische Auseinander- 
setzung des Wissenschaftlers mit erkenntnistheoretischen Fragen im Sinne Kants für unverzichtbar. Er sagt:

«Kants Philosophie beabsichtigte nicht, die Zahl unserer Kenntnisse durch das reine Denken zu vermehren, denn ihr oberster Satz war, dass alle Erkenntniss der Wirklichkeit aus der Erfahrung geschöpft werden müsse, sondern sie beabsichtigte nur, die Quellen unseres Wissens und den Grad seiner Berechtigung zu untersuchen, ein Geschäft, welches immer der Philosophie verbleiben wird, und dem sich kein Zeitalter ungestraft wird entziehen können.» ${ }^{15}$

«Wissenschaftlichkeit» im Erkennen und theoretisch begründeten Handeln ist nicht schon durch den Nachweis einer bestimmten Methode erreicht, auf die man sich beruft. Vom Wissenschaftler fordert v. Helmholtz vielmehr, dass er das Verhältnis von Methode und Gegenstand systematisch reflektiert, d.h., dass er Erkenntnistheorie betreibt; denn erkenntnistheoretische Reflexionen haben nicht weniger als naturwissenschaftliche Untersuchungen ihren realen Gegenstand : das geistige Tätigsein des Menschen. So stellt v. Helmholtz fest, dass es sich bei der Aufgabe, «die Leistungsfähigkeit unseres Denkvermögens zu untersuchen», ebenso «um eine Reihe thatsächlicher Fragen» ${ }^{16}$ handelt.

Von Helmholtz anerkennt damit einerseits die Wissenschaftlichkeit der Philosophie selbst und billigt dieser andererseits eine wissenschaftskonstituierende Funktion in anderen Wissenschaften zu. Er begrenzt aber zugleich den Aufgabenbereich der Philosophie auf den der Wissenschaftstheorie, eine Reduktion, der Kant nicht zugestimmt hätte, wenn sich dieser Aspekt philosophischen Denkens auch in der Kritik der reinen Vernunft in der Unterscheidung der Begriffe Organon und Kanon wiederfindet. Von daher ist v. Helmholtz' Erkenntnistheorie vor allem darauf hin zu befragen, ob es möglich ist, Philosophie auf Wissenschaftstheorie zu beschränken; denn Philosophie bedeutet für Kant nicht nur Methodenkritik. «Denken» ist Tätigkeit des Verstandes, die Selbstbewusstsein ermöglicht, in der sich der Anspruch des Menschen auf Selbstsein und freie Selbstbestimmung manifestiert. Wird Denken aber nicht in seiner Freiheit konstituierenden Bedeutung verstanden, sondern auf die Funktion der Methodenbegründung reduziert, so ist zumindest fraglich, inwiefern eine medizinische Wissenschaftstheorie über die Frage der Zweckmässigkeit der Mittel hinaus die Grundfrage medizinischer Wissenschaft kritisch beurteilen kann, die lautet: Inwiefern ist es möglich, technisches Handeln am Menschen mit dessen Anspruch freier Selbstbestimmung zu vermitteln? 


\section{Von Helmholtz' «empiristische Theorie»}

Von Helmholtz bestimmt in seiner Erkenntnistheorie die Sinnesempfindungen als Zeichen, «deren besondere Art ganz von unserer Organisation abhängt». Und im Sinne von Kants grundlegender Unterscheidung von Ding an sich und Erscheinungsding fügt er hinzu: «... so sind sie doch nicht als leerer Schein zu verwerfen, sondern sie sind eben Zeichen von Etwas». ${ }^{17}$

Empirie gründet in der Sinnesempfindung und wird zum selbstbewussten Erkennen in der Synthese von sinnlicher Wahrnehmung und Denken. In diesem Verhältnis werden Sinneseindrücke dem Menschen bewusst und durch die logisch-diskursive Verstandestätigkeit zugleich auf grössere Wirklichkeitsentsprechung hin korrigiert. Der logische Schluss, «dass das gleiche Object, unter gleichen Umständen zur Einwirkung kommend, das gleiche Zeichen hervorruft, und dass also ungleiche Zeichen immer ungleicher Einwirkung entsprechen, ${ }^{18}$ ermöglicht die erkennende Annäherung an das Gesetz, nach dem Gegenstände erscheinen und sich verändern. Damit ist wiederum das Naturgesetz, das als «die Abbildung der Gesetzmässigkeit in den Vorgängen der wirklichen Welt» ${ }^{19}$ bestimmt wird, primäres Erkenntnisziel der empiristischen Theorie:

\footnotetext{
«Jedes Naturgesetz sagt aus, dass auf Vorbedingungen, die in gewisser Beziehung gleich sind, immer Folgen eintreten, die in gewisser anderer Beziehung gleich sind. Da Gleiches in unserer Empfindungswelt durch gleiche Zeichen angezeigt wird, so wird der naturgesetzlichen Folge gleicher Wirkungen auf gleiche Ursachen auch eine ebenso regelmässige Folge im Gebiete unserer Empfindungen entsprechen.» ${ }^{20}$
}

Das Erkennen der Naturgesetze begreift v. Helmholtz als einen Lernprozess. So wird «Sehen» möglich, indem der Mensch lernt, «die Vorstellung eines gewissen Gegenstandes mit gewissen Empfindungen zu verknüpfen, welche wir wahrnehmen. ${ }^{21}$ Lernen ist ein induktiver Vorgang. In wiederholten Empfindungen entsteht die Vorstellung von einem Gegenstand, und sich immer wieder neu bildende Vorstellungen führen zu einem Bild von der Wirklichkeit des Gegenstandes und der Gesetzmässigkeit seines Erscheinens; denn es ist «das Gesetzmässige, was sich am regelmässigsten gleichartig wiederholt, während das zufällig Wechselnde verwischt wird.» ${ }^{22}$ V. Helmholtz ist sich bewusst, dass «weder die Lückenhaftigkeit unseres Wissens, noch die Natur der Inductionsschlüsse» die «unbedingte Behauptung» ${ }^{23}$ der Naturgesetze gestattet. Induktive Verfahren beruhen auf dem «Vertrauen, dass ein bisher beobachtetes gesetzliches Verhalten sich auch in allen noch 
nicht zur Beobachtung gekommenen Fällen bewähren werde.» ${ }^{24}$ Bewährung aber bedeutet, dass ein durch Induktion aufgestelltes Gesetz sich in der deduktiven Vorhersage von Gegebenheiten je neu zu verifizieren hat, ohne dass die Möglichkeit, einmal falsifiziert zu werden, mit letzter Gewissheit ausgeschlossen werden kann. Es entstehen also Hypothesen als logische Zusammenhänge induktiver und deduktiver Sätze. ${ }^{25}$ Die durch Induktion abgeleiteten und als wirklichkeitsentsprechend vorausgesetzten Naturgesetze ermöglichen die deduktive Vorhersage von Gegebenheiten, die wiederum die logische Grundlage für die Ableitung von Handlungsanweisungen bildet. Diese sagen, auf welche Weise bestimmte Gegebenheiten zu verändern sind, damit unter der Voraussetzung, dass ein bestimmtes Naturgesetz der Wirklichkeit entspricht, die Gegebenheiten eintreten, die im Handeln erzeugt werden sollen. Sinn und Bewährung der Hypothese werden nach v. Helmholtz erst im Handeln offenkundig:

«Unzweifelhaft ist die realistische Hypothese die einfachste, die wir bilden können, geprüft und bestätigt in ausserordentlich weiten Kreisen der Anwendung, scharf definiert in allen Einzelbestimmungen und deshalb ausserordentlich brauchbar und fruchtbar als Grundlage für das Handeln.» ${ }^{26}$

Was hier möglicherweise als Anspruch auf Letztgültigkeit und damit dem Charakter hypothetischen Wissens widersprechend erscheint, versucht v. Helmholtz abzumildern, indem er betont, Hypothesen tun so, «als ob die [...] angenommene Welt der stofflichen Dinge wirklich bestände»; und weiter sagt er: «Aber über dieses 〈als ob〉 kommen wir nicht hinweg [...]; nothwendige Wahrheit dürfen wir ihr [der Hypothese] nicht zuschreiben.» ${ }^{27}$ Von Helmholtz ist sich mit Kant darin einig, dass durch Induktionsschlüsse die Differenz von Erscheinungsding und Ding an sich nicht aufgehoben und die Wirklichkeit selbst im Erkennen einzelner Gegebenheiten nicht letztgültig bestimmt werden kann. Es gibt keine abschliessende Vermittlung von denkendem Ich und Wirklichkeit. Von Helmholtz' Erkenntnistheorie unterscheidet sich damit eindeutig von positivistischen Positionen. Zudem bestimmt v. Helmholtz die Kausalität im Sinne Kants (und gegen die Auffassungen der englischen Empiristen) als Denkform a priori, die nicht aus der Erfahrung abgeleitet ist.

Von Helmholtz bestimmt Kausalität aber nicht nur als Kategorie; er geht noch einen Schritt weiter und setzt die Kategorie der Kausalität gleich mit einem «regulativen Prinzip» ${ }^{28}$. Was bedeutet dies für die Gesamtkonzep- 
tion einer Erkenntnistheorie? Die Bildung von Hypothesen, die Aussagen machen über die Beziehungen zwischen einzelnen Phänomenen in der Natur, ist nach Kant möglich in der logischen Voraussetzung der regulativen Idee der Natur als eines teleologischen Ordnungszusammenhangs. «Natur» ist demnach kein empirischer Begriff; denn die Ganzheit der Natur ist dem Menschen nicht gegeben. Sie ist vielmehr die Gesamthypothese, in deren vorausgesetztem Sinn das erkennende Ich Natur denkt und Einzelhypothesen bildet. Von Helmholtz anerkennt die Hypothesenstruktur wissenschaftlicher Sätze in bezug auf Einzelphänomene; hinsichtlich der Gesamtheit der Natur aber glaubt er, die hypothetische Begrenztheit unseres Erkennens überwinden zu können. So behauptet er: «Was wir aber unzweideutig und als Thatsache ohne hypothetische Unterschiebung finden können, ist das Gesetzliche in der Erscheinung.» ${ }^{29}$ Damit werden wissenschaftliche Sätze über Naturgesetze nicht mehr, wie Kant es wollte, als Hypothesen unter der regulativen Idee eines teleologischen Ordnungszusammenhangs verstanden. Von Helmholtz erhebt vielmehr Gesetzeshypothesen der Naturerkenntnis zu einer Erkenntnis der Natur an sich.

Daraus wird nun der Widerspruch verständlich, wenn v. Helmholtz die Kausalität zwar einerseits als kategoriale Denkform im Sinne Kants begreift, andererseits aber Kants Schluss, dass wohl etwas in der Wirklichkeit sein muss, um erscheinen zu können, dazu benutzt, die Kausalität als durchgehendes Gesetz nicht nur der gedachten Welt, sondern der wirklichen Ordnung vorauszusetzen.

Beweis dafür ist v. Helmholtz der Vorgang der Empfindung selbst, den er als Verhältnis von Ursache und Wirkung zwischen Gegenstand und Sinnen bestimmt:

\begin{abstract}
«Von dem ersten Schritt an, wo wir vor uns weilende Objecte im Raume vertheilt wahrnehmen, ist die Wahrnehmung das Anerkennen einer gesetzlichen Verbindung zwischen unseren Bewegungen und den dabei auftretenden Empfindungen. Schon die ersten elementaren Vorstellungen enthalten also in sich ein Denken und gehen nach den Gesetzen des Denkens vor sich.» ${ }^{30}$
\end{abstract}

Und an anderer Stelle schreibt v. Helmholtz:

"Auf welche Weise sind wir denn nun zuerst aus der Welt der Empfindungen unserer Nerven hinübergelangt in die Welt der Wirklichkeit? Offenbar nur durch einen Schluss; wir müssen die Gegenwart äusserer Objecte als Ursache unserer Nervenerregung voraussetzen; denn es kann keine Wirkung ohne Ursache sein.» ${ }^{31}$ 


\section{Theorie und Praxis}

Stellt man die Frage, warum v. Helmholtz' eigener Grundsatz: «Unwürdig eines wissenschaftlich sein wollenden Denkers aber ist es, wenn er den hypothetischen Ursprung seiner Sätze vergisst» ${ }^{32}$, für das Wissen um Naturgesetze nicht gelten soll, wird man einerseits auf das Verhältnis von Denkgesetz und Naturgesetz und andererseits auf das von Theorie und Praxis verwiesen. Von Helmholtz geht einerseits aus von der apriorischen Geltung der Kausalität als Denkgesetz, in dessen Voraussetzung der Erkennende Natur interpretiert. Andererseits erkennt er in der «Gesetzmässigkeit ... die Bedingung der Begreifbarkeit» und schliesst aus der regelmässigen Wiederkehr von Erscheinungen auf eine gesetzmässige Ordnung der Natur, die deren Denkbarkeit bis hin zur «vollkommenen Begreifbarkeit der Welt» ermöglicht :

«Wir können in einer Welt leben, in der jedes Atom von jedem anderen verschieden wäre, und wo es nichts Ruhendes gäbe. Da würde keinerlei Regelmässigkeit zu finden sein, und unsere Denkthätigkeit müsste ruhen.» ${ }^{33}$

Damit endet aber von Helmholtz' Versuch, das Verhältnis von Denkgesetz und Naturgesetz zu bestimmen, in einem Zirkelschluss. In diesem wird einerseits Welt aufgrund des kategorialen Denkvermögens des Menschen als universale Kausalordnung begriffen, und andererseits wird eine reale, kausal geordnete Ganzheit der Natur als Möglichkeitsbedingung für die Sinnhaftigkeit, Naturvorgänge kausal zu interpretieren, vorausgesetzt. Diesen Zirkelschluss versucht von Helmholtz durch einen weiteren Begründungsversuch aufzulösen. So sieht er in der Möglichkeit, kausalanalytisches Wissen technisch anzuwenden, die letztgültige Bestätigung für das reale Vorhandensein einer durchgehend kausal bestimmten Naturordnung. Er sagt: «Für die Anwendbarkeit des Causalgesetzes haben wir aber keine weitere Bürgschaft, als seinen Erfolg.» ${ }^{34}$ Damit stellt aber auch das Verhältnis von Theorie und Praxis bei v. Helmholtz einen Zirkelschluss dar. Einerseits soll kausalanalytisches Wissen in einem linearen Anwendungsverhältnis technisches Handeln rechtfertigen: «Das Begreifen [...] ist die Methode, mittels deren unser Denken die Welt sich unterwirft, die Thatsachen ordnet, die Zukunft voraus bestimmt» ${ }^{35}$; und andererseits wird der technische Erfolg, das tatsächliche Eintreten vorhergesagter Gegebenheiten im technischen Handeln, als Bestätigung der in der Theorie vorausgesetzten Kausalordnung der Natur angesehen. Von Helmholtz schreibt dazu : 
«Das erste Product des denkenden Begreifens der Erscheinung ist das Gesetzliche. Haben wir es so weit rein ausgeschieden, seine Bedingungen so vollständig und sicher abgegrenzt und zugleich so allgemein gefasst, dass für alle möglicher Weise eintretenden Fälle der Erfolg eindeutig bestimmt ist, und wir gleichzeitig die Ueberzeugung gewinnen, es habe sich bewährt und werde sich bewähren in aller Zeit und in allen Fällen: dann erkennen wir es als ein unabhängig von unserem Vorstellen Bestehendes an und nennen es die Ursache, d.h. das hinter dem Wechsel ursprünglich Bleibende und Bestehende.» ${ }^{36}$

Von Helmholtz' primäres Erkenntnisinteresse ist das Naturgesetz als theoretische Absicherung technischen Handelns in einem linearen Anwendungsverhältnis von kausalanalytischem Wissen und technischer Verfügbarkeit. Darin spiegelt sich einerseits der für die zweite Hälfte des 19. Jahrhunderts typische Glaube an die technische Beherrschbarkeit der Natur wider; andererseits ist für v. Helmholtz auch das handlungstheoretische Grundproblem der Medizin interesseleitend, dass medizinische Handlungen, einmal ausgeführt, nicht wieder rückgängig gemacht werden können, während zu ihrer handlungstheoretischen Orientierung lediglich hypothetische, nicht eindeutig verifizierbare Sätze zur Verfügung stehen, die nicht in der Lage sind, die Folgen konkreten Handelns bereits im Vorfeld der Theorie mit Gewissheit vorherzusagen :

«Noch nothwendiger sind die Hypothesen für das Handeln, weil man nicht immer zuwarten kann, bis eine gesicherte wissenschaftliche Entscheidung erreicht ist, sondern sich nach der Wahrscheinlichkeit... entscheiden muss.» ${ }^{37}$

Angesichts der Entscheidungsnotwendigkeit in der Medizin hält es von Helmholtz für eine ethische Pflicht, die theoretische Wissensbildung voranzutreiben. So frage sich der Arzt, «... ob man selbst Alles gethan habe, was man zur Abwehr des Verhängnisses hätte thun können, und ob die Wissenschaft auch wohl alle Kenntnisse und Hülfsmittel vorbereitet habe, die sie hätte vorbereiten sollen, um zu wissen, dass erkenntnistheoretische Fragen über die Methodik der Wissenschaft auch eine bedrängende Schwere und furchtbare praktische Tragweite erlangen können.» ${ }^{38}$

\section{Medizin als Naturwissenschaft}

Ausgehend von der Darstellung der «empiristischen Theorie» kann nun das Verhältnis von Medizin und Naturwissenschaft, wie es v. Helmholtz versteht, bestimmt werden. Die Möglichkeit, die Medizin als Naturwissenschaft zu begründen, setzt voraus, dass die Differenz von anorganischer und organischer Natur methodisch aufgehoben werden kann. Dazu versucht v. Helm- 
holtz zu zeigen, dass Naturgesetze über die Grenzen dieser heuristischen Unterscheidung hinaus gelten.

In seiner Rede, «Ueber das Ziel und die Fortschritte der Naturwissenschaften», legte v. Helmholtz 1869 vor der Naturforscherversammlung in Innsbruck dar, dass genau diese Bedingung durch das Gesetz von der Erhaltung der Kraft erfüllt wird. Dieses war bereits 1842 von dem schwäbischen Arzt Julius Robert Mayer (1814-1878) formuliert worden und sagt, «dass die Quantität der in dem Naturganzen vorhandenen wirkungsfähigen Kraft unveränderlich sei, weder vermehrt noch vermindert werden könne.» ${ }^{39}$ Die Geltung dieses Gesetzes für die gesamte Natur ist darin begründet, dass es bei gleichzeitiger Erhaltung der Kraft deren Umwandlungsmöglichkeit in den verschiedenen Naturvorgängen aufzeigt. Für die Äquivalenz der Formen wirkender Kräfte gab bereits Robert Mayer bestimmte Messwerte an, die durch Joule präzisiert wurden. Demnach besteht eine Vergleichbarkeit zwischen Wärme und mechanischer Arbeitskraft, deren Verhältnis in Zahlen ausgedrückt werden kann:

«Eine bestimmte Wärmemenge kann in eine bestimmte Menge von Arbeit verwandelt werden; diese Arbeitsmenge kann aber auch in Wärme, und zwar genau in dieselbe Wärmemenge zurückverwandelt werden, aus der sie entstanden ist; in mechanischer Beziehung sind beide einander äquivalent. Die Wärme ist eine neue Form, in welcher ein Quantum von Arbeitskraft erscheinen kann.» ${ }^{40}$

Von Helmholtz erkennt im Gesetz von der Erhaltung der Kraft die Möglichkeit, Natur in allen ihren wechselnden Erscheinungen und Energieformen als Einheit gesetzmässig wirkender Kräfte zu begreifen. Er sagt:

«Alle Veränderung in der Natur besteht darin, dass die Arbeitskraft ihre Form und ihren Ort wechselt, ohne dass ihre Quantität verändert wird. Das Weltall besitzt ein für alle Mal einen Schatz von Arbeitskraft, der durch keinen Wechsel der Erscheinungen verändert, vermehrt oder vermindert werden kann und der alle in ihm vorgehenden Veränderungen unterhält.» ${ }^{42}$

Dabei ist Kraft als Wärme erzeugende Bewegung zugleich «das objektivierte Gesetz der Wirkung». ${ }^{42}$ Das bedeutet, v. Helmholtz entwickelt eine gegenständliche Vorstellung von den Gesetzmässigkeiten der Natur, die als verschiedene und zugleich vergleichbare Formen wirkender Kräfte wahrgenommen, gemessen und mathematisch dargestellt werden können:

«Wenn wir uns vergewissern können, dass die Bedingungen eingetreten sind, unter denen das Gesetz zu wirken hat, so müssen wir auch den Erfolg eintreten sehen ohne Willkür, ohne Wahl, ohne unser Zutun, mit einer die Dinge der Aussenwelt ebenso gut, wie unser 
Wahrnehmen, zwingenden Notwendigkeit. So tritt uns das Gesetz als eine objektive Macht entgegen, und demgemäss nennen wir es Kraft.» ${ }^{43}$

Bemerkenswert ist, dass hier bereits v. Helmholtz das Verhältnis von Naturnotwendigkeit und Entscheidungsfreiheit des Menschen anspricht, das er in einer späteren Bemerkung noch verdeutlicht:

«Insofern wir dann das Gesetz als ein unsere Wahrnehmung und den Ablauf der Naturprozesse Zwingendes, als eine unserem Willen gleichwerthige Macht anerkennen, nennen wir es $\langle\mathrm{Kraft}\rangle »{ }^{44}$

Auf der Grundlage des Gesetzes von der Erhaltung der Kraft bestimmt nun v. Helmholtz das Verhältnis von Physik und Physiologie und begründet Medizin als Naturwissenschaft, indem er sagt:

\begin{abstract}
«Ist aber das Gesetz von der Erhaltung der Kraft auch für die lebenden Wesen gültig, so folgt daraus, dass die physikalischen und chemischen Kräfte der zum Aufbau ihres Körpers verwendeten Stoffe ohne Unterbrechung und ohne Willkür fortdauernd tätig sind, und dass ihre strenge Gesetzlichkeit in keinem Augenblick durchbrochen wird.

Die Physiologie musste sich entschliessen, mit einer unbedingten Gesetzlichkeit der Naturkräfte auch in der Erforschung der Lebensvorgänge zu rechnen; sie musste Ernst machen mit der Verfolgung der physikalischen und chemischen Prozesse, die innerhalb der Organismen vor sich gehen.» ${ }^{45}$
\end{abstract}

Damit ist gesagt, Physik und Physiologie seien zwei Wissenschaften, deren Erkenntnisinteresse auf dieselbe Naturgesetzlichkeit ausgerichtet ist. Sofern aber die Differenz von anorganischer und organischer Natur in einer durchgehenden Kausalordnung aufgehoben ist, muss «das Endziel der Naturwissenschaften $[\ldots]$, die allen anderen Veränderungen zugrunde liegenden Bewegungen und deren Triebkräfte zu finden, also sich in Mechanik aufzulösen» ${ }^{46}$, auch für die Medizin gelten. Medizinisches Handeln hat sich demnach aufzulösen in die kausalanalytisch begründete technische Veränderung empirischer Gegebenheiten am Menschen.

\title{
Schlussfolgerung
}

Aus der Darstellung der v. Helmholtzschen Erkenntnistheorie und der daraus abgeleiteten wissenschaftstheoretischen Bestimmung, inwiefern sich in seinem Denken Hinwendung der Medizin zum Wissenschaftsverständnis der Physik vollzieht, ergibt sich besonders eine Schlussfolgerung, die nun noch erörtert werden soll.

Von Helmholtz' Erkenntnis- und Wissenschaftstheorie ist gekennzeichnet von einem Widerspruch. Dieser rührt daher, dass v. Helmholtz einerseits 
eine Erkenntnistheorie vertritt, die an Kants transzendentaler Methode orientiert ist. Von daher entgeht von Helmholtz' Denken dem Fallstrick des Positivismus, der empirische Gegebenheiten als die Wirklichkeit der Dinge zu bestimmen versucht.

Während von Helmholtz aber für die empirische Erkenntnis Kants Differenz von Erscheinungsding und Ding an sich anerkennt und mit guten Gründen verteidigt, dass Kausalität kein empirischer Begriff ist, gelangt er dennoch zu einem ausschliesslich technischen Praxisverständnis der Medizin, das von einem auf Funktionalität reduzierten Menschenbild ausgeht bzw. dieses als die ausschliessliche Wirklichkeit des Menschen behauptet. Der auf diese Weise vorherbestimmte Konflikt, in den von Helmholtz die praktische Medizin und deren theoretische Begründung letztlich hineinführt, besteht nun nicht darin, dass auch Funktionalität und technische Verfügbarkeit zur Wirklichkeit des Menschen gehören. Es ist vielmehr das als Grundproblem medizinischer Wissenschaftstheorie erkannte Verhältnis von technischem Handeln am Menschen und dessen Anspruch auf freie Selbstbestimmung, in dem v. Helmholtz einer deterministischen, die Freiheit des Menschen negierenden Bestimmung letztlich nicht mehr ausweichen kann.

Kant hatte dieses Problem klar erkannt als Verhältnis von kausal bestimmter Naturnotwendigkeit und Kausalität aus Freiheit, und rettete die Freiheit, indem er streng unterschied zwischen der Kategorie Kausalität und der regulativen Idee eines teleologischen Ordnungszusammenhangs, in dessen Voraussetzung darüber entschieden wird, inwiefern es sinnvoll ist, bei der Interpretation bestimmter Phänomene die Kausalität als kategoriale Denkform auf die Verhältnisse in der Natur anzuwenden. Bestimmt von dem Interesse, eindeutige Handlungsanweisungen aus kausalanalytischem Wissen abzuleiten, setzt v. Helmholtz hingegen die Kategorie der Kausalität gleich mit der regulativen Idee einer in sich kausal geordneten Gesamtwirklichkeit der Natur, und erhebt diese zu einem Über-Gegebenen, das er in den Naturgesetzen vollständig begreifen zu können glaubt. 


\section{Anmerkungen}

1 Hermann von Helmholtz: Vorträge und Reden. 2 Bde. 3. Aufl. Braunschweig 1884, hier Bd.1, S. IX.

2 Zitiert in Herbert Hörz; Siegfried Wollgast: Hermann von Helmholtz und Emil du BoisReymond. In: Christa Kirsten (Hrsg.): Dokumente einer Freundschaft: Briefwechsel zwischen Hermann von Helmholtz und Emil du Bois-Reymond 1846-1894. Berlin 1986, hier S. 25.

3 Vgl. Hermann von Helmholtz: Vorträge und Reden. 2 Bde. 4. Aufl. Braunschweig 18911896, hier Bd.1, S. 17.

4 Vgl. Walter Israel: Substanzbegriff und Energieproblem in der modernen Physik: Eine erkenntnistheoretische Studie über Robert Mayer, Hermann von Helmholtz und Max Planck. Berlin 1921, S.53-56.

5 Vgl. Hörz und Wollgast (wie Anm.2), S. 54.

6 Vgl. Leo Königsberger: Hermann von Helmholtz' Untersuchungen über die Grundlagen der Mathematik und Mechanik. Heidelberg 1895, hier S.5.

7 Vgl. Hörz und Wollgast (wie Anm.2), S.49.

8 Von Helmholtz (wie Anm.3), Bd.1, S. 8.

9 Vgl. Leo Königsberger: Über Helmholtz's Bruchstück eines Entwurfs betitelt «Naturforscher-Rede». Heidelberg 1910 (Sitzungsberichte der Heidelberger Akademie der Wissenschaften, math.-naturwiss. Klasse 14), hier S. 7.

10 Von Helmholtz (wie Anm. 3), S.241.

11 Von Helmholtz (wie Anm. 3), Bd.2, S. 240.

12 Vgl. v. Helmholtz (wie Anm. 3), Bd. 1, S. 99.

13 Von Helmholtz (wie Anm. 3), Bd.1, S. 99.

14 Von Helmholtz (wie Anm. 3), Bd.2, S. 218.

15 Von Helmholtz (wie Anm. 3), Bd. 1, S. 88.

16 Vgl. v. Helmholtz (wie Anm. 3), Bd.1, S.16.

17 Vgl. v. Helmholtz (wie Anm. 3), Bd.2, S. 223.

18 Ebd., S.222.

19 Ebd., S.222.

20 Ebd., S.222.

21 Von Helmholtz (wie Anm. 3), Bd.1, S.114.

22 Von Helmholtz (wie Anm. 3), Bd.2, S. 232.

23 Ebd., S. 243.

24 Ebd., S. 238-239.

25 Ebd., S. 238-239.

26 Ebd., S.239.

27 Ebd., S. 239.

28 Ebd., S.243.

29 Ebd., S. 240.

30 Ebd., S. 240.

31 Von Helmholtz (wie Anm. 3), Bd. 1, S. 115-116.

32 Von Helmholtz (wie Anm. 3), Bd.2, S. 239.

33 Ebd., S. 243. 
34 Ebd., S.243.

35 Ebd., S.243.

36 Ebd., S.241.

37 Ebd., S. 239.

38 Hermann v. Helmholtz: Das Denken in der Medicin. 2. Aufl. Berlin 1878, hier S.6.

39 Von Helmholtz (wie Anm.3), Bd. 1, S. 192.

40 Ebd., S. 217-218.

41 Ebd., S.227.

42 Vgl. Hermann v. Helmholtz: Über die Entwicklungsgeschichte der neueren Naturwissenschaften. In: Hansjochem Autrum (Hrsg.): Von der Naturforschung zur Naturwissenschaft. Vorträge, gehalten auf Versammlungen der Gesellschaft deutscher Naturforscher und Ärzte (1828-1958). Berlin 1987, hier S. 37.

43 Ebd., S.36.

44 Von Helmholtz (wie Anm. 3), Bd.2, S. 241.

45 Von Helmholtz (wie Anm.42), S. 49.

46 Ebd., S.40.

Prof. Dr. J. N. Neumann

Institut für Geschichte der Medizin

Krausenstrasse 14

D-06097 Halle/Saale 